\title{
Salmonella EM FRANGOS E A CONTAMINAÇÃO CRUZADA POR MEIO DE
} SUPERFICIES DE CORTE EM COZINHAS DOMÉSTICAS

\author{
Vanessa Mendonça Soares ${ }^{1}$ \\ Juliano Gonçalves Pereira ${ }^{2}$ \\ Lorena Natalino Haber Garcia ${ }^{3}$ \\ José Paes de Almeida Nogueira Pinto ${ }^{2}$
}

\begin{abstract}
RESUMO
Dentre os patógenos responsáveis por doenças transmitidas por alimentos, Salmonella é considerado o de maior importância para a saúde pública, sendo o sorotipo $S$. Enteritidis o mais frequentemente envolvido nos casos de infecção em humanos. Esta revisão aborda aspectos relacionados à epidemiologia da salmonelose e Salmonella, assim como a importância do frango cru como fonte de contaminação para superfícies de corte utilizadas nas cozinhas domésticas para a manipulação de alimentos e sua consequente disseminação por meio de contaminação cruzada.
\end{abstract}

Palavras-chave: contaminação cruzada, Salmonella, superfícies de corte.

\section{Salmonella IN POULTRY AND CROSS-CONTAMINATION THROUGH CUTTING BOARDS IN DOMESTIC KITCHENS}

\begin{abstract}
Among the pathogens responsible for causing food-borne diseases, Salmonella is considered the most important for public health, and the serotype $S$. Enteritidis is the most involved in cases of human infection. This literature review focuses on aspects related to the epidemiology of salmonellosis and on Salmonella, as well as on the importance of raw chicken as a source of contamination of cutting boards used in domestic kitchens for food handling and its subsequent spread through cross contamination.
\end{abstract}

Key words: cross contamination, cutting boards, Salmonella Enteritidis.

\section{Salmonella EN POLLOS Y LA CONTAMINACIÓN CRUZADA A TRAVÉS DE SUPERFICIES DE CORTE EN COCINAS DOMÉSTICAS}

\section{RESUMEN}

Entre los agentes patógenos causantes de enfermedades transmitidas por alimentos, Salmonella es considerado el más importante para la salud pública, y el serotipo $S$. Enteritidis lo más involucrado en los casos de infección humana. Esta revisión se centra en aspectos relacionados con la epidemiología de la salmonelosis y en Salmonella, así como en la importancia de pollo crudo como fuente de contaminación de las superficies de corte utilizadas en las cocinas domésticas para la manipulación de los alimentos y su posterior propagación a través de la contaminación cruzada.

\footnotetext{
1 Universidade Estadual Paulista "Júlio de Mesquita Filho" - UNESP

2 Universidade Estadual Paulista "Júlio de Mesquita Filho - UNESP, Departamento de Produção Animal e Medicina

Veterinária Preventiva. Correspondência juliano.pereira@unesp.br

3 Universidade Federal de Viçosa, Departamento de Veterinária.
}

Soares VM, Pereira JG, Garcia LNH, Pinto JPAN. Salmonella em frangos e a contaminação cruzada por meio de superfícies de corte em cozinhas domésticas Vet. e Zootec. 2020; 27: 001-012. 
Palabras-clave: contaminación cruzada, Salmonella, superficies de corte.

\section{INTRODUÇÃO}

Uma das doenças de origem alimentar mais frequente no mundo é a salmonelose, uma gastrenterite causada pelo consumo de alimentos contaminados por Salmonella spp. Do ponto de vista epidemiológico, o sorotipo Enteritidis tem ganhado maior destaque nas últimas décadas, uma vez que os dados o associam como principal causa de salmonelose humana. A contaminação por $S$. Enteritidis se dá, principalmente, pelo consumo de produtos avícolas, entre eles a carne de frango. O potencial de risco associado ao consumo destes produtos vem sendo demonstrado por diversos estudos, os quais apontam altas prevalências do patógeno neste alimento em especial.

Como tais produtos são amplamente adquiridos pelos consumidores domésticos, configura-se um risco de ocorrência da enfermidade entre os mesmos. A disseminação do patógeno nas cozinhas domésticas ocorre, especialmente, por um evento denominado contaminação cruzada, caracterizado pela transferência de células de Salmonella da carne crua de frango naturalmente contaminada, via superfícies de corte, para alimentos prontos para o consumo.

Durante a manipulação e preparo dos alimentos em cozinhas domésticas, a aplicação de procedimentos corretos de higienização é de grande importância para a redução da contaminação microbiológica de superfícies de corte, diminuindo, assim, o risco de contaminação cruzada, com reflexos na saúde dos consumidores. Os procedimentos normalmente utilizados pelos consumidores para a limpeza de superfícies, inadequados em determinadas situações, devem ser avaliados para se conhecer o grau de exposição a que estes podem estar expostos.

Esta revisão tem como objetivo abordar particularidades relacionadas com contaminação cruzada envolvendo carcaças de frangos contaminadas com $S$. Enteritidis, contribuir com aspectos específicos relacionados com a inocuidade das refeições que são preparadas e consumidas no âmbito doméstico e, deste modo, oferecer subsídios para ações de saúde pública que visam à proteção da saúde dos consumidores.

\section{DESENVOLVIMENTO}

\section{Salmonelose e Salmonella spp.}

Uma das doenças de origem alimentar mais frequente no mundo é a salmonelose (1), decorrente, em $95 \%$ dos casos, da ingestão de alimentos contaminados com células viáveis de sorotipos de Salmonella $(2,3)$. Os sintomas caracterizam-se por dor abdominal, calafrios, febre, vômito, desidratação e dores de cabeça, sendo que o período de incubação pode variar de 5 a 72 horas, frequentemente ocorrendo entre 12 a 36 horas. A recuperação normalmente se dá de 1 a 4 dias após o aparecimento dos sintomas, sendo que a maioria dos casos é autolimitante (4).

A possibilidade de uma pessoa adoecer, a gravidade e a duração da infecção podem estar relacionadas a diversos fatores que incluem desde o tipo de alimento, o número de células ingeridas, a virulência e o sorotipo de Salmonella até a condição do sistema imune do indivíduo $(1,4,5)$. No tocante à dose mínima infectante, relatos demonstram que normalmente são necessárias mais de $10^{6}$ UFC de Salmonella/g ou $\mathrm{mL}$ do alimento para gerar um quadro de infecção, embora doses baixas, isto é, de 1 a 100 células já tenham sido relatadas (6).

Do ponto de vista epidemiológico, embora os casos de salmonelose possam ser causados por diversos sorotipos, $S$. Enteritidis é reconhecido como o principal responsável por gerar 
infecção em humanos $(7,8)$. No mundo, surtos de salmonelose humana causados por este sorotipo começaram a aumentar de forma acentuada a partir do final da década de 80 e, no Brasil, esse aumento foi observado por volta de 1993, situando $S$. Enteritidis como importante problema econômico e de saúde pública, sendo responsável por mais de $60 \%$ dos sorotipos isolados no Instituto Adolfo Lutz (9).

Dados recentes referem que, entre os anos 2009 e 2015, os americanos confirmaram, com auxílio de exames laboratoriais, 100.939 casos de doenças de origem alimentar, sendo que $30 \%$ deles (23.662) estavam relacionados com Salmonella. Este dado estatístico se manteve constante no ano de 2016, dos 839 surtos de origem animal confirmados durante o período, $33 \%$ deles eram representados por Salmonella, sendo Enteritidis o sorovar mais comum reportado durante o ano (10).

No último relatório apresentado pelas autoridades de segurança alimentar da União Europeia, referente ao ano de 2016, foram confirmados 94.530 casos de salmonelose; deste modo, a taxa de notificação média para União Europeia foi de 20,4 casos/100.000 habitantes, com uma ampla variação entre os países membros da comunidade. A menor incidência ocorreu em Portugal, com 3,6, e a maior na população da República Checa, com 110/100.000 habitantes. $S$. Enteritidis foi o principal sorotipo envolvido em casos de infecção por Salmonella em humanos na União Europeia, cerca de $59 \%$ de 44.462 casos confirmados da enfermidade (11).

Em relação ao Brasil, durante o período de 2000 a 2017 foram notificados 12.619 surtos de doença de origem alimentar, com o envolvimento de 238.909 doentes. Embora não tenha ocorrido a identificação do agente etiológico em todos os surtos, em 2.593 deles, Salmonella foi o micro-organismo mais comumente isolado, correspondendo a $34,1 \%$ (884) do total dos surtos identificados (12).

Greig e Ravel (13) analisaram dados de 4.093 surtos de doença de origem alimentar que ocorreram em diversos países do mundo entre 1988 e 2007, e verificaram que 46,9\% deles foram causados por Salmonella. Outra informação destacada foi a atribuição de $24,1 \%$ do total dos surtos à $S$. Enteritidis, situando o sorotipo como o principal patógeno de origem alimentar.

Conforme Freitas Neto (14), a análise da prevalência ao longo do tempo demonstra que existe uma dinâmica entre os diferentes sorovares e, em determinados períodos, existe um equilíbrio em favor de um único sorovar mais prevalente. Entre 2004 e 2010, S. Enteritidis permaneceu como o sorovar mais prevalente em amostras da cadeia produtiva de frangos, estando presente em $47,75 \%$ dos isolamentos realizados de 2004 a 2008 e em 9,86\% nos de 2009 a 2010.

\section{Salmonella em frangos}

O consumo de carne de frangos e ovos contaminados por Salmonella é uma das principais causas da salmonelose humana $(15,16)$. Segundo Freitas et al. (17), a presença deste patógeno em frangos caracteriza este alimento como inseguro para o consumo.

Descrições de surtos de salmonelose relacionados ao consumo de frango são encontradas com frequência na literatura. Nos EUA, por exemplo, dados relativos ao ano de 2016 revelaram que $12 \%$ dos casos de salmonelose humana estavam relacionados ao consumo de frango (10, 18). Frango contaminado com Salmonella também foi reportado como a principal causa de surtos de doença de origem alimentar na Inglaterra entre os anos de 1992 e 2008 (19).

Segundo Greig e Ravel (13), de 4.093 surtos de doenças transmitidas por alimentos ocorridos entre 1988 e 2007 no mundo, 34\% deles estavam associados ao consumo de carne de frango e, destes, em $45 \%$ identificou-se o sorotipo $S$. Enteritidis.

Os animais são reservatórios naturais de Salmonella, sendo frequente o seu encontro no trato gastrintestinal de várias espécies, embora as aves sejam consideradas o principal reservatório (3). Em relação aos frangos de corte, quando estes são contaminados ainda na

Soares VM, Pereira JG, Garcia LNH, Pinto JPAN. Salmonella em frangos e a contaminação cruzada por meio de superfícies de corte em cozinhas domésticas Vet. e Zootec. 2020; 27: 001-012. 
granja, acabam se tornando portadores e a disseminação da Salmonella para outras carcaças pode ocorrer pela contaminação fecal durante as operações de abate (20). Em outras palavras, o processo industrial para a obtenção de carcaças de frango e seus respectivos cortes para consumo são fatores de risco para sua contaminação, principalmente durante os procedimentos de evisceração, resfriamento, embalagem e transporte (16).

As aves de criação industrial contaminam-se por Salmonella, geralmente, pela transmissão vertical via ovo ou no seu ambiente de criação pela ração, roedores e outros animais. A presença do agente em ração, farinha de carne, de pena ou vísceras, secreções de roedores, insetos e na cama do aviário aumenta e sustenta índices de contaminação cada vez maiores nos plantéis avícolas $(20,21)$.

No que diz respeito a Salmonella em amostras de carcaças e cortes de frango, numerosos estudos têm avaliado sua presença e demonstram ocorrências variando de 7 a 48 \% (22).

A ocorrência de Salmonella em carcaças de frango no ano de 2016, nos Estados Unidos, foi de $10 \%$ (10). Já na União Europeia, um monitoramento em 25.276 carcaças de frango mostrou uma positividade de $6,39 \%$ ao longo da cadeia de produção, situando este produto como um dos principais veiculadores de Salmonella dentre alimentos diversos (11).

A análise de 150 amostras de frangos congelados obtidos do varejo da cidade de Jaboticabal, São Paulo, constatou uma contaminação de 32 \% das amostras por Salmonella, sendo que $60 \%$ dos sorotipos identificados eram $S$. Enteritidis (23). Outro estudo brasileiro, concluiu que houve uma alta incidência de $S$. Enteritidis em $57,7 \%$ e $84 \%$ em aves reprodutoras e frangos de corte, respectivamente (24).

É importante ressaltar que, embora a contaminação por Salmonella em frangos seja normalmente expressa na forma de presença/ausência, do ponto de vista de saúde pública, dados a respeito das contagens do patógeno no alimento são mais significativos, já que podem ser empregados em avaliações de risco microbiológico. A elaboração deste tipo de avaliação, ao logo da cadeia de produção de frangos, é importante para a redução de Salmonella no produto acabado (21).

Dentro deste contexto, uma pesquisa conduzida nos Estados Unidos em 60 carcaças de frango encontrou uma contagem média de $20 \mathrm{UFC} /$ carcaça (25). Na Austrália, Pointon et al. (26) avaliaram amostras de 549 carcaças e 310 cortes de frango, tendo registrado contagens de $2,5 \mathrm{UFC} / \mathrm{cm}^{2}$ e $3,8 \mathrm{UFC} / \mathrm{cm}^{2}$, respectivamente. As contagens médias de 220 filés de peito foram de $779 \mathrm{UFC} /$ filé em estudo realizado na Holanda (27).

\section{Salmonella e o ambiente doméstico}

As doenças transmitidas por alimentos são definidas como enfermidades infecciosas ou tóxicas causadas pelo consumo de alimento ou água contaminados. Na atualidade, são consideradas um dos maiores problemas mundiais de saúde pública. A estimativa anual é de que são afetados por episódios de doenças alimentares ou relacionados a alimentos 130 milhões de europeus, 2,1 a 3,5 milhões de ingleses e galeses, 76 milhões de americanos e 4,7 milhões de australianos (20).

Em relação ao local de ocorrência destes surtos, o ambiente doméstico tem um papel importante. Até $87 \%$ deles estão associados com alimentos preparados ou consumidos em residências nos países da Europa, Austrália, Nova Zelândia, Estados Unidos e Canadá (28). Dados relativos a alguns países europeus ilustram essa variação entre surtos ocorridos no ambiente doméstico: 32\% na Dinamarca, 7\% na Hungria, 4\% na Romênia, 24\% na Finlândia, $52 \%$ na Polônia e $10 \%$ nos EUA (29).

No Brasil, entre 2000 e 2017, dos surtos que tiveram o local de ocorrência identificado, $36,4 \%$ deles aconteceram em ambiente doméstico (12). Dos 1148 surtos notificados no estado do Rio Grande do Sul entre os anos de 2000 e 2014, 45,6\% também tiveram o ambiente

Soares VM, Pereira JG, Garcia LNH, Pinto JPAN. Salmonella em frangos e a contaminação cruzada por meio de superfícies de corte em cozinhas domésticas Vet. e Zootec. 2020; 27: 001-012. 
doméstico como local de ocorrência (30). Entretanto, a proporção de surtos que ocorrem nas residências provavelmente seja maior do que o reportado, uma vez que são menos identificados pelas autoridades de saúde pública, já que acometem um ou poucos indivíduos e, geralmente, causam sintomas brandos, fazendo com que os envolvidos não busquem auxílio médico (31, 32).

Dos diversos micro-organismos patogênicos que são continuamente introduzidos nas residências pelas pessoas, alimentos, animais de estimação, insetos, água de abastecimento contaminada e ar, Salmonella tem sido o patógeno mais implicado em surtos de origem alimentar relacionados com domicílios $(21,29,33)$. No Brasil, isto também tem se verificado, e os dados igualmente indicam Salmonella como o principal agente e o ambiente doméstico como o local de maior ocorrência de surtos $(9,34,35,36)$.

Especificamente em relação aos alimentos, a introdução de Salmonella no ambiente doméstico pode ocorrer especialmente pela carne de frango contaminada (36). Assim, a associação entre Salmonella e a alta frequência com que o patógeno pode ser encontrado, ilustram o risco potencial associado com a manipulação deste alimento no ambiente doméstico, já que dentre os produtos crus vendidos para o público, ele é o que tem a maior probabilidade de estar contaminado, quando comparado a outros (37).

Entre os diversos fatores que contribuem para a ocorrência de surtos de doença de origem alimentar, as práticas inadequadas de manipulação dos alimentos pelos consumidores têm papel fundamental $(30,38)$. Estima-se que somente elas sejam responsáveis por $40-60 \%$ dos casos de doenças alimentares originárias nas residências (39).

A observação de consumidores ou a simulação de suas práticas no preparo de alimentos para a obtenção de dados a respeito da presença de Salmonella na cozinha, de sua sobrevivência e transferência aos alimentos e das práticas de limpeza associadas demonstraram que a manipulação inadequada de alimentos pelos consumidores e a falta de higiene aumentam o risco de ocorrência da enfermidade nos moradores (40).

Pesquisa realizada para avaliar o nível de conhecimento dos consumidores a respeito de perigos microbiológicos de origem alimentar e práticas de manipulação de alimentos nos Estados Unidos divulgou dados interessantes. O levantamento envolvendo 1.620 americanos que utilizam cozinhas domésticas mostrou que $80,2 \%$ deles já tinham ouvido falar de Salmonella. Quando foram indagados a respeito do alimento relacionado com a veiculação do patógeno, 53,7\% conheciam esta informação. Estas mesmas pessoas foram as que mais comumente relataram possuir práticas adequadas de higiene e manipulação de alimentos como, por exemplo, lavar mãos e superfícies de corte após o contato com carne de frango crua, quando comparadas às pessoas que não foram capazes de relacionar o alimento envolvido na veiculação do agente. É importante ressaltar também que a maior proporção destas pessoas $(64,3 \%)$ tinha mais de 12 anos de estudo (41).

No entanto, uma análise criteriosa destes dados deve ser feita, uma vez que, embora manipuladores de alimentos em ambiente doméstico possam ter um bom nível de conhecimento sobre práticas seguras de manipulação de alimentos e relatem seu emprego, na prática, suas atitudes podem demonstrar o contrário $(40,42)$. Assim, fica evidente que, para minimizar e prevenir a disseminação de doenças de origem alimentar por parte dos consumidores no ambiente doméstico, conhecimento, consciência e atitude em relação às boas práticas de manipulação de alimentos são necessárias e essenciais. Tais metas são alcançadas através da educação e promoção de mudanças comportamentais no público em geral (30). Deve-se considerar, no entanto, que os consumidores nem sempre associam práticas de manipulação de alimentos no ambiente doméstico com doenças de origem alimentar. Desta forma, torna-se difícil convencê-los a realizar mudanças de comportamento em relação à manipulação de alimentos (43)

Soares VM, Pereira JG, Garcia LNH, Pinto JPAN. Salmonella em frangos e a contaminação cruzada por meio de superfícies de corte em cozinhas domésticas Vet. e Zootec. 2020; 27: 001-012. 
No caso específico de Salmonella, a manipulação inadequada de alimentos, temperaturas de abuso, cozimento inadequado, utilização de ingredientes crus contaminados e a contaminação cruzada perfazem as práticas fortemente associadas a surtos da enfermidade em humanos (3).

\section{Salmonella e a contaminação cruzada de alimentos prontos para o consumo via superfícies de corte}

A contaminação cruzada durante o preparo dos alimentos é um fator que contribui significativamente para a ocorrência de surtos de origem alimentar $(22,44,45)$. Dados apontam que ela tem sido implicada em um terço dos eventos (46), embora este valor seja provavelmente subestimado (37).

Contaminação cruzada é definida como a transmissão de patógenos de fontes naturalmente contaminadas para um produto acabado. Um exemplo é a transferência de bactérias de carcaças de frango naturalmente contaminadas, via superfícies não higienizadas, para alimentos prontos para o consumo, tais como saladas $(22,28,45)$. Este evento em particular é considerado uma das principais formas de exposição humana à Salmonella proveniente da carne de frango (40). A possibilidade de uma infecção ocorrer devido ao contato com superfícies contaminadas é ratificada pelas evidências que apontam que a ingestão ou contato com pequenos números de micro-organismos patogênicos podem ser suficientes para causar a infecção $(4,7,44)$.

Contaminação cruzada via superfícies inanimadas durante o preparo doméstico de frangos contaminados foram demonstradas por Dewit et al. (47). Estudos recentes mostram que superfícies de corte podem ser contaminadas com números elevados de Salmonella $(>1.000$ UFC) durante o preparo de frango contaminado $(6,34)$. Esta elevada transferência pode ser explicada, possivelmente, pelo fato de que células de Salmonella Enteritidis possuem estruturas superficiais, como fímbrias, que facilitam sua adesão em superfícies inanimadas $(48,49)$.

Adicionalmente, em relação às carcaças de aves, tem-se a preocupação de que o líquido remanescente, presente nas embalagens e na própria carne crua de frango, possa contaminar as superfícies de corte com patógenos e, a partir daí haver transferência das células para os alimentos que não sofrerão processo de cozimento antes de serem consumidos $(50,51)$. Superfícies de corte de uso doméstico são empregadas nas cozinhas em uma diversidade de tarefas durante o preparo de alimentos. Para ilustrar essa situação, uma pesquisa revelou que 30 a $71 \%$ dos consumidores usam a mesma superfície de corte para preparar carne crua e outros alimentos, como saladas (8). Logo, uma limpeza inadequada após a manipulação de carne de frango pode exercer um papel fundamental na contaminação por Salmonella, salientando-se que o patógeno pode sobreviver por pelo menos 24 horas em superfícies encontradas nas cozinhas $(40,46)$.

Pesquisadores têm mostrado o perfil dos consumidores em relação à contaminação cruzada e os comportamentos de higiene relacionados às superfícies de corte. Pelos resultados, verificou-se que até $75 \%$ dos consumidores carecem de familiaridade com o termo contaminação cruzada e os riscos a ela associados (21). Altekruse et al. (52) entrevistaram 19.356 pessoas, comprovando que $19 \%$ dos consumidores não lavam rotineiramente superfícies de corte com sabão ou água sanitária após cortarem carne crua ou frango. Pesquisa conduzida pelo FDA (Food and Drug Administration) apontou que uma em cada cinco pessoas entrevistadas respondeu não lavar superfícies de corte após o contato com carne de frango crua (53).

Um estudo avaliando a taxa de transferência de $S$. enterica de frangos para alface por meio de superfícies de plástico e faca em diversos cenários de limpeza verificou que quando os utensílios não eram higienizados após a sua utilização, a transferência foi de $1,25 \%$ do frango

Soares VM, Pereira JG, Garcia LNH, Pinto JPAN. Salmonella em frangos e a contaminação cruzada por meio de superfícies de corte em cozinhas domésticas Vet. e Zootec. 2020; 27: 001-012. 
para o plástico e de $45,62 \%$ do plástico e faca para a alface (38). Outro estudo demonstrou que a limpeza inadequada das superfícies onde o frango cru contaminado com Salmonella foi manipulado foi responsável pela contaminação de $81 \%$ das saladas preparadas (21).

Além das superfícies serem foco de contaminação para alimentos que entram em contato com estas, existe a questão do tipo de material utilizado para a produção destes artefatos e sua facilidade de higienização. A maior facilidade para a remoção de restos de alimentos e microorganismos, em alguns tipos de superfícies, as caracterizam como mais seguras por serem mais higiênicas (8), e apresentarem menor risco de contaminação cruzada (7).

Acredita-se que as superfícies de madeira sejam mais difíceis de higienizar e sanitizar do que de as de plástico, pois as primeiras apresentam porosidades (50). Contudo, Ak et al. (54) demonstraram, em estudo que procurou verificar possíveis diferenças no processo de descontaminação de superfícies de corte, que mais bactérias eram recuperadas de superfícies produzidas com material plástico do que naquelas de madeira, concluindo que, quando usados procedimentos de higienização razoáveis, artefatos de madeira poderiam ser utilizados com segurança em cozinhas domésticas. Posteriormente, resultados de estudo realizado por Gough e Dodd (55), com objetivos semelhantes e conduzidos com $S$. Typhimurium, mostraram que a madeira apresentava um potencial de risco para contaminação cruzada maior que o plástico.

Outras superfícies também têm sido alvo de estudos. Moore et al. (56) pesquisaram a taxa de recuperação de $S$. Typhimurium a partir da fórmica, aço inoxidável, polipropileno e madeira, e verificaram que as taxas de recuperação foram superiores na fórmica e no aço inoxidável em relação ao polipropileno e à madeira, sendo que, neste estudo, o processo de limpeza não foi considerado.

Durante a manipulação e preparo dos alimentos, o objetivo da aplicação de procedimentos de higienização é reduzir a contaminação microbiológica para níveis seguros em superfícies que representam um risco de contaminação cruzada, consequentemente reduzindo o risco de ocorrência deste evento $(35,53,54)$. Dos métodos utilizados para a limpeza de superfícies, o emprego apenas de sabão e água nem sempre resulta na descontaminação de superfícies $(6,55$, $56,57,58)$. Por outro lado, embora os desinfetantes possam ser mais efetivos na redução da população de Salmonella nas cozinhas domésticas (59), poucos estudos são encontrados a respeito de sua eficiência em diferentes tipos de superfícies de corte com vistas a prevenção da contaminação cruzada durante a manipulação de alimentos no ambiente doméstico.

Deve-se salientar que a compreensão do modelo de transferência de bactérias para alimentos durante os eventos de contaminação cruzada ainda é um grande desafio (21).

\section{CONCLUSÃO}

A contaminação cruzada em nível doméstico tem um papel fundamental na perda da inocuidade dos alimentos em função das superfícies de corte utilizadas pelos consumidores durante a manipulação de frango cru contaminado com Salmonella, especialmente quando nenhum método de higienização é aplicado. Os consumidores devem ser orientados em relação à adoção de práticas que evitem a disseminação dos patógenos em suas cozinhas, garantindo a segurança de suas refeições e a não ocorrência de doenças transmitidas por alimentos.

\section{REFERÊNCIAS}

1. Bollaerts K, Aerts M, Faes C, Grijspeerdt K, Dewulf J, Mintiens K. Human salmonellosis: estimation of dose-illness from outbreak data. Risk Analysis. 2008;28:427-440.

2. Frazier WC, Westhof DC. Food Microbiology. 4 ed. New York: McGraw-Hill International Edition; 1988.

Soares VM, Pereira JG, Garcia LNH, Pinto JPAN. Salmonella em frangos e a contaminação cruzada por meio de superfícies de corte em cozinhas domésticas Vet. e Zootec. 2020; 27: 001-012. 
3. Jay JM. Microbiologia de alimentos. 6 ed., Porto Alegre: Artmed; 2005.

4. Cliver DO. Foodborne Diseases. Califórnia: Academic Press; 1990.

5. Franco BDGM, Landgraf M. Microbiologia de alimentos. São Paulo: Atheneu; 2005.

6. Bari M, Ukuku D. Foodborne Pathogens and Food Safety. Boca Raton: CRC Press; 2016.

7. Germano PML, Miguel M, Miguel O, Germano MIS. Prevenção e controle das toxinfecções de origem alimentar. Higiene Alimentar. 1993;7(27): 6-11.

8. Floweres FL. Salmonella. Food Technology.1988;42(4):182-185.

9. Kottwitz LBM, Oliveira TCRM, Alcocer I, Farah SMSS, Abrahão WSM, Rodrigues DP. Avaliação epidemiológica de surtos de salmonelose ocorridos no período de 1999 a 2008 no Estado do Paraná, Brasil. Acta Scientiarum Health Sciences. 2010;32:9-15.

10. Centers for Disease Control and Prevention (CDC). Surveillance for Foodborne Disease Outbreaks United States, 2016, Annual Report. Atlanta, Georgia: U.S. Department of Health and Human Services, CDC, 2018. [acesso em 08 Jan 2018] Disponível em: < https://www.cdc.gov/fdoss/pdf/2016_FoodBorneOutbreaks_508.pdf>

11. European Food Safety Authority (EFSA). The European Union summary report on trends and sources of zoonoses, zoonotic agents and food-borne outbreaks in 2016, EFSA Journal. 2017;15(12):21-56.

12. Brasil Surtos de Doenças Transmitidas por Alimentos no Brasil. Brasília, 2018. [cited 2018 Jan] Available from:

http://portalarquivos2.saude.gov.br/images/pdf/2018/julho/02/Apresentacao-SurtosDTA-Junho-2018.pdfs.

13. Greig JD, Ravel A. Analysis of foodborne outbreak data reported internationally for source attribution. International Journal of Food Microbiology. 2009;130:77-87.

14. Freitas Neto OC, Penha Filho RAC, Barrow P, Berchieri Junior A. Sources of human nontyphoid salmonellosis: a review. Revista Brasileira de Ciência Avícola. 2010;12(1):1-11.

15. Back A. Manual de doenças de aves. Cascavel: Mercolab; 2002.

16. Duarte DAM, Ribeiro AR, Vasconcelos AMM, Santos SB, Silva JVD, Andrade PLA, Falcão LSPCA. Occurrence of Salmonella spp. in broiler chicken carcasses and their susceptibility to antimicrobial agents. Brazilian Journal of Microbiology. 2009;40:569573.

17. Freitas CG, Santana AP, Silva PHC, Gonçalves VSP, Barros MAF, Torres FAG, et al. PCR multiplex for detection of Salmonella Enteritidis, Typhi and Typhimurium and occurrence in poultry meat. International Journal of Food Microbiology. 2010;139:15-22. 
18. Barba FJ, Koubaa M, Prado-Silva L, Orlien V, Sant'Ana AS. Mild processing applied to the inactivation of the main foodborne bacterial pathogens: A review. Trends in Food Science \& Technology. 2017;66:20-35.

19. Gormley FJ, Ittle CLL, Rawal N, Illesp IAG, Adak GK. A 17-year review of foodborne outbreaks: describing the continuing decline in England and Wales (1992-2008) Epidemiology \& Infection. 2010;139:688-699.

20. Mead G, Lammerding AM, Cox N, Doyle MP, Humbert F, Kulikovskiy A. Scientific and technical factors affecting the setting of Salmonella criteria for raw poultry: a global perspective. Journal of Food Protection. 2010;73:1566-1590.

21. Redmond EC, Griffith CJ. Consumer food handling in the home: a review of food safety studies. Journal of Food Protection. 2003;66:130-161

22. Luber P. Cross-contamination versus undercooking of poultry meat or eggs — which risks need to be managed first?. International Journal of Food Microbiology. 2009;134:21-28.

23. Santos DMS, Junior AB, Fernandes SA, Tavechio AT, Amaral LA. Salmonella em carcaças de frango congeladas. Pesquisa Veterinária Brasileira. 2000;20:39-42.

24. Kanashiro AMI; Stoppa GFZ; Cardoso ALSP; Tessari ENC; Castro AGM. Serovars of Salmonella spp. isolated from broiler chicken and commercial breeders in diverse regions in Brazil from July 1997 to December 2004. Brazilian Journal of Poultry Science. 2005;7(3):195-198.

25. Brichta-Harhay DM, Arthur TM, Koohmaraie M. Enumeration of Salmonella from poultry carcass rinses via direct plating methods. Letters in Applied Microbiology. 2008;46:186-191.

26. Pointon A, Sexton M, Dowsett P, Saptura T, Kiemeier A, Lorimer M, et al. A Baseline survey of the microbiological quality of chicken portions and carcasses at retail in two Australian states (2005 to 2006). Journal of Food Protection. 2008;70:1123-1134.

27. Straver JM, Janssen AFW, Linnemann AR, Van Boekel MAJS, Beumer RR, Zwietering MH. Number of Salmonella on chicken breast fillet at retail level and its implications for public health risk. Journal of Food Protection. 2007;70:2045-2055.

28. Asselt ED, De Jong AEI, De Jonge R, Nauta, MJ. Cross-contamination in the kitchen: estimation of transfer rates for cutting boards, hands and knives. Journal of Applied Microbiology. 2008;105:1392-1401.

29. Klein LR, Bisognin RP, Figueiredo DMS. Estudo do perfil epidemiológico dos surtos de doenças de transmissão hídrica e alimentar no Rio Grande do Sul: uma revisão dos registros no estado. Revista Brasileira de Geografia Médica e da Saúde. 2017;13(25):4864.

30. Gorman, R.; Bloomfield, S.; Adley, C.C. A study of cross-contamination of food-borne pathogens in the domestic kitchen in the Republic of Ireland. International Journal of Food Microbiology. 2002;76:143-150.

Soares VM, Pereira JG, Garcia LNH, Pinto JPAN. Salmonella em frangos e a contaminação cruzada por meio de superfícies de corte em cozinhas domésticas Vet. e Zootec. 2020; 27: 001-012. 
31. Ferreira JAF. Panorama das Doenças Transmitidas por Alimentos no Brasil entre 2000 e 2015. Dissertação (Mestrado) Faculdade de Saúde Pública da Universidade de São Paulo, São Paulo, 2017. Disponível em: < https:/www.teses.usp.br/teses/disponiveis/6/6138/tde11052017-165548/publico/JessicaDeAragaoFreireFerreraREVISADA.pdf >.

32. Marins BR, Tancredi RCP, Gemal AL. Segurança alimentar no contexto da vigilância sanitária: reflexões e práticas. Rio de Janeiro: EPSJV, 2014. 287p.

33. Welker CAD, Both JMC, Longaray SM, Haas S, Soeiro MLT, Ramos RC. Análise microbiológica dos alimentos envolvidos em surtos de doenças transmitidas por alimentos (DTA) ocorridos no estado do Rio Grande do Sul, Brasil. Revista Brasileira de Biociências. 2010;8:44-48.

34. Melo ES, Amorim WR, Pinheiro REE, Corrêa PGN, Carvalho SMR, Santos ARSS, Barros DS, Oliveira ETAC, Mendes CA, Sousa FV. Doenças transmitidas por alimentos e principais agentes bacterianos envolvidos em surtos no Brasil: revisão. Pubvet. 2018;12(10):1-9.

35. Cardoso ALSP, Tessari ENC. Salmonella enteritidis em aves e na saúde pública: revisão de literatura. Revista científica eletrônica de medicina veterinária. 2013;11(21).

36. Shinohara NKS, Barros VB, Jimenez SMC, Machado EdeCL, Dutra RAF, Lima FJL. Salmonella spp., importante agente patogênico veiculado em alimentos. Ciênc. saúde coletiva. 2008; 13(5): 1675-1683. Disponível em: $<\mathrm{http}: / /$ www.scielo.br/scielo.php?script=sci_arttext\&pid=S141381232008000500031\&1 ng=en .

37. Kennedy J, Nolan A, Gibney S, O'Brien S, McMahon MAS, McKenzie K, Healy B, McDowell D, Fanning S, Wall PG. Determinants of cross-contamination during home food preparation. British Food Journal. 2011;113(2):280-297.

38. Frewer LJ, Fischer ARH. Consumer perceptions of risks from food. Handbook of hygiene control in the food industry. Cambridge, UK: Woodhead publishing. 2005:103-119.

39. Ravishankar S, Zhu L, Jaroni D. Assessing the cross contamination and transfer rates of Salmonella enteric from chicken to lettuce under different food-handling scenarios. Food Microbiology. 2010;27:1-4.

40. De Jong AEI, Verhoeff-Bakkenes L, Nauta MJ, De Jong R. Cross-contamination in the kitchen: effect of hygiene measures. Journal of Applied Microbiology. 2008;105:615624.

41. Asselt ED, Fischer A, Jong AEI, Nauta MJ, Jong R. Cooking practices in the kitchenobserved versus predicted behavior. Risk Analysis. 2009;29:533-540.

42. Griffith CJ, Price P, Peters AC, Clayton DA. An evaluation of food handlers knowledge, belief and attitudes about food safety and its interpretation using social cognition models. Food Standards Agency, London, 2001. 
43. Ravarotto L, Crovato S, Mantovani C, D’Este F, Pinto A, Mascarello G. Reducing microbiological risk in the kitchen: piloting consensus conference methodology as a communication strategy. Journal of Risk Research. 2016;19(7):934-950.

44. Jacob C, Mathiasen L, Powell D. Designing effective messages for microbial food safety hazards. Food Control. 2010;21(1):1-6.

45. Taché J, Carpentier B. Hygiene in the home kitchen: Changes in behaviour and impact of key microbiological hazard control measures. Food Control. 2014;35(214):392-400.

46. Borruso PA, Quinlan JJ. Prevalence of pathogens and indicator organisms in home kitchens and correlation with unsafe food handling practices and conditions. Journal of Food Protection. 2017;80(4):590-597.

47. Dewitt JC, Brokhuizer G, Kamplemacher EH Cross-contamination during preparation of frozen chicken in the kitchen. Journal of Hygiene. 1979;83:27-32.

48. Sternisa M, Mozina SS, Levstek S, Kukec A, Raspor P, Jevsnik M. Food safety knowledge, self-reported practices and attitude of poultry meat handling among Slovenian consumers. British Food Journal. 2018;120(6):1344-1357.

49. Woodward MJ, Sojka M, Sprogings KA, Humphrey TJ. The role of SEF14 and SEF17 $\sigma$ fimbriae in the adherence of Salmonella enterica serotype Enteritidis in inanimate surfaces. Journal of Medical Microbiology. 2000;49:481-487.

50. Aviat F, Gerhards C, Rodriguez-Jerez J, Michel V, Bayon IL, Ismail R, Federighi M. Microbial Safety of Wood in Contact with Food: A Review. Comprehensive Reviews in Food Science and Food Safety. 2016;15:491-505.

51. Wilcock A, Ball B. Practical Food Safety: Contemporary Issues and Future Directions. UK (Chichester). [s.n]. 2014:11-29.

52. Altekruse SF, Street DA, Fein SB, Levy AS. Consumer knowledge of foodborne microbial hazards and food-handling practices. Journal of Food Protection. 1996;59(3):287-294.

53. Cliver DO. Cutting boards in Salmonella cross-contamination. Journal of AOAC International. 2006;89:538-542.

54. Ak NO, Cliver DO, Kaspar CW. Decontamination of plastic and wooden cutting boards for kitchen use. Journal of Food Protection. 1994;57:23-30.

55. Gough NL, Dodd CER. The survival and disinfection of Salmonella typhimurium on chopping board surfaces of wood and plastic. Food Control. 1998;9:363-368.

56. Moore G, Blair IS, Mcdowell DA. Recovery and transfer of Salmonella Typhimurium from four different domestic food contact surfaces. Journal of Food Protection. 2007;70:2273-2280. 
57. Soares VM, Pereira JG, Viana C, Izidoro TB, Bersot LS, Pinto JPAN. Transfer of Salmonella Enteritidis to four types of surfaces after cleaning procedures and crosscontamination to tomatoes. Food Microbiology. 2012;30(2):453-456.

58. Verran J, Redfern J. Testing surface cleanability in food processing. 2 ed., Oxford: Elsevier; 2016.

59. Siani H, Maillard JY. Best practice in healthcare environment decontamination. European Journal of Clinical Microbiology. 2015;34(1):1-11.

Recebido em: 23/09/2019 Aceito em: 21/02/2020 